\title{
Tratamiento endourológico de estenosis ureterales. Estudio experimental comparativo
}

\author{
F. Soria Gálvez, L.Á. Rioja Sanz*, M. Blas Marin*, Mª . E. Durán Flores, J. Usón Gargallo \\ Unidad de Endoscopia. Centro de Cirugía de Mínima Invasión. Cáceres. \\ *Servicio de Urología. Hospital Miguel Servet. Zaragoza \\ Actas Urol Esp 2005; 29 (3): 296-304
}

\begin{abstract}
RESUMEN
TRATAMIENTO ENDOUROLÓGICO DE ESTENOSIS URETERALES. ESTUDIO EXPERIMENTAL COMPARATIVO

Objetivo: Los objetivos de este estudio son la comparación de la eficacia y la eficiencia entre dos técnicas endourológicas para el tratamiento de estenosis ureterales en modelo animal, así como la valoración histológica de la cicatrización ureteral.

Material y métodos: Se emplearon 10 animales de la sp. porcina. A los que se les sometió a un modelo experimental de estenosis ureteral. Transcurridas 4 semanas se realiza el diagnóstico de la obstrucción urinaria. Dividimos a los animales en 2 grupos:

-Grupo I. (5 animales), endoureterotomía con balón hasta la ruptura ureteral.

-Grupo II (5 animales), tratamiento mediante Acucise ${ }^{\circledR}$.

Se disponen catéteres doble pigtail durante 3 semanas. Realizándose el seguimiento a las 4 semanas de la extracción de los mismos.

Resultados: Todos los animales del estudio mostraron signos de obstrucción urinaria tras 4 semanas de la ligadura ureteral. De los 10 animales, uno de cada grupo necesitó una segunda aplicación de la técnica en la misma sesión. Encontramos en el Grupo I, la migración en sentido vesical de un catéter ureteral que provoca la restenosis. Tras la valoración de los resultados determinamos que el porcentaje de éxito es de un $80 \%$ en el grupo I y de un $100 \%$ en el II.

Conclusiones: A la vista de los resultados obtenidos en nuestro estudio las dos técnicas se muestran efectivas, aunque el Acucise ${ }^{\circledR}$ se mostró más eficiente. No encontramos evidencias histológicas que avalen los estudios de cicatrización de Davis tras la endoureterotomía.
\end{abstract}

Palabras clave: Uréter. Porcino. Endoureterotomía. Modelo experimental. Estenosis. Ultrasonidos endoluminales.

\section{ABSTRACT}

ENDOUROLOGIC TREATMENT OF URETERAL STRICTURES. EXPERIMENTAL COMPARATIVE STUDY

Objectives: to compare efficacy and efficiency of two different endourological therapies for ureteral stricture, and to evaluate pathological reactions of the ureters following both endourological techniques.

Material and methods: Ten pigs underwent experimental induction of ureteral stricture. Four weeks later, ureteral strictures were demonstrated by imaging techniques. Animals were divided in two groups, according to the received therapy:

-Group I. (5 pigs), endoballoon rupture endoureterotomy.

-Group II (5 pigs), Acucise ${ }^{\circledR}$ balloon endoureterotomy.

Ureteral stents were placed for 3 weeks following endoureterotomy. Animals were followed up four weeks after ureteral stents retrieval.

Results. In all cases, ureteral stricture was proved four weeks after model induction. In one case from each group, it was needed a second balloon dilatation to achieve complete endoureterotomy. Leading to ureteral restenosis, stent migration occurred in one animal from group I. Success was achieved in $80 \%$ of cases from group I, and $100 \%$ of cases from group II.

Conclusions. Our results suggest that both endourological therapies are effective. Nevertheless, a higher efficiency was proved with Acucise ${ }^{\circledR}$ endoureterotomy. Our pathological evidences do not support Davis's studies on ureteral healing following endoureterotomy.

Keywords: Ureter. Swine. Endoureterotomy. Experimental model. Stricture. Endoluminal ultrasound.

Este trabajo ha sido subvencionado por la Fundación para la Investigación en Urología (Asociación Española de Urología) Beca Martín de Castellanos 2003, patrocinada por Merck Sharp Dohme. "Estudio comparativo de la necesidad de empleo de stents ureterales tras endopielotomía retrógrada en modelo animal obstrucción ureteropiélica". 
$\mathrm{L}$ as estenosis ureterales resultan de multitud de causas, incluyendo procedimientos endoscópicos urológicos, avance de litiasis, radioterapia y cirugía laparoscópica o convencional (urológica, vascular y ginecológica) ${ }^{1}$. El tratamiento de las estenosis ureterales ha evolucionado mucho durante los últimos 25 años, desde los tratamientos quirúrgicos clásicos mediante cirugía abierta hasta las modernas técnicas basadas en la cirugía de mínima invasión para la dilatación de la zona estenótica. Las técnicas endourológicas tienden a reducir el daño quirúrgico, la morbilidad, la administración de analgésicos, el tiempo de intervención y de convalecencia ${ }^{2}$.

La introducción del catéter de balón por Grüntzig et al en 1974, proporcionó el primer medio eficaz de dilatación selectiva de áreas estenóticas de vasos sanguíneos ${ }^{3}$. Esto estimuló su aplicación en diferentes estructuras orgánicas tubulares no vasculares, sobre todo en el sistema gastrointestinal, uretra y uréter.

Tras los estudios de Davis en 1943, surgió en la década de los 80 la aplicación de su técnica dentro de la Endourología ${ }^{4}$. Epingoud et al en 1980, utilizó por primera vez el catéter de balón para dilatar estenosis del aparato urinario. El uso del balón de dilatación como método exclusivo de tratamiento de las estenosis ureterales vía endourológica se inicia en 1981 en un paciente que sufrió la ligadura del uréter con catgut por causas iatrogénicas ${ }^{5}$.

Los abordajes endourológicos para el tratamiento de estenosis ureterales se han convertido en la actualidad en una efectiva alternativa frente a la cirugía convencional. Aunque existe una mayor experiencia de estas técnicas en el tratamiento de la unión pieloureteral, también es posible su uso en estenosis más caudales ${ }^{6,7}$.

Los objetivos de este estudio son pues la comparación de la eficacia y la eficiencia entre dos técnicas endourológicas para el tratamiento de estenosis ureterales vía retrógrada en modelo animal, asî como la valoración histológica de la cicatrización ureteral tras el tratamiento endourológico.

\section{MATERIAL Y MÉTODOS}

Se seleccionan animales de la especie porcina para la realización de este trabajo por sus semejanzas con la especie humana en el tracto uri- nario superior ${ }^{8}$. Para la realización de este estudio empleamos diez hembras sanas de la especie porcina con pesos comprendidos entre 35 y 40 Kg. Todos los procedimientos se realizan bajo anestesia general inhalatoria y con control analgésico en su recuperación postoperatoria (1 $\mathrm{mg} / \mathrm{Kg}$ Ketorolaco).

El estudio se efectúa tras la aprobación del protocolo por parte del Comité de ética de experimentación animal del Centro de Cirugía de Mínima Invasión de Cáceres. Tras el periodo preceptivo de adaptación y cuarentena de los animales se inicia el estudio.

Planteamos el estudio en tres fases:

Fase I: Creación del modelo de estenosis ureteral benigna

Tras la anestesia del animal, se extrae una muestra de sangre para la determinación de la urea y creatinina séricas. Procedemos posteriormente a la valoración nefrosonográfica de ambos riñones siguiendo las pautas de la SFU (Society for Fetal Urology).

Completamos una exploración cistoureteroscópica mediante un ureteroscopio flexible, que nos permite la extracción de la muestra de orina para su cultivo bacteriológico y la exploración del uréter derecho. A continuación alojamos una guía, para el manejo endourológico de la vía urinaria, retirando el ureteroscopio. Empleamos el riñón y uréter izquierdos como control. También realizamos una cistografía para descartar reflujo vesicoureteral que interfiera con los resultados del estudio. A continuación llevamos a cabo una urografía excretora, donde valoramos tanto aspectos morfológicos como funcionales.

A través de la guía alojada en el uréter, ascendemos un catéter de marcas radio-opacas y completamos una ureteropielografía retrógrada (RUPG), para la medición del diámetro ureteral interno en la zona de estudio (a $2 \mathrm{~cm}$ de la unión pieloureteral, tomando como referencia la articulación intervertebral $\mathrm{L}_{4}-\mathrm{L}_{5}$ ), de este modo además del diámetro interno obtenemos imágenes del trayecto ureteral, descartando estrecheces o caliectasia previa. Aprovechando este abordaje procedemos a la valoración mediante ultrasonografía endoluminal (ELUS) del trayecto ureteral (ClearView- Boston Scientific). 
Finalizada esta exploración alojamos en el uréter derecho un catéter angiográfico Cobra II de $4 \mathrm{Fr}$ para que sirva de tutor en el modelo de estenosis ureteral.

El modelo de obstrucción urinaria parcial del uréter proximal, necesita tres trocares, dos de 10 $\mathrm{mm}$ y uno de $5 \mathrm{~mm}$. El primero para la óptica, otro para la pinza de disección y el último para las tijeras y el portaagujas laparoscópico. La óptica se coloca a nivel de la cicatriz umbilical, los otros dos puertos adelantados a este se ubican a $6 \mathrm{~cm}$ de la línea media abdominal, separados entre ellos 20 cms y formando un triángulo entre los tres puertos. Se sitúa al cerdo en decúbito lateral izquierdo para desplazar las vísceras intrabdominales medialmente y de este modo conformar un campo quirúrgico amplio.

Identificamos el lugar exacto donde se realizará la estenosis gracias a la combinación de laparoscopia y fluoroscopia, comenzando la disección laparoscópica del uréter y creando una ventana de $360^{\circ}$ alrededor de este, liberando 2 $\mathrm{cm}$ del recorrido ureteral, para posteriormente ligar el uréter con Caprosyn ${ }^{\circledR} 3 / 0$, evitando la obstrucción completa gracias al catéter alojado en el interior del uréter. Esta ligadura se realiza mediante anudado manual laparoscópico con la ayuda de un disector y del portaagujas laparoscópico (Fig. 1).

Finalizada la ligadura laparoscópica del uréter, se chequea la estrechez ureteral mediante la inyección de contraste yodado diluido a través del catéter alojado en el uréter, se retira el gas del

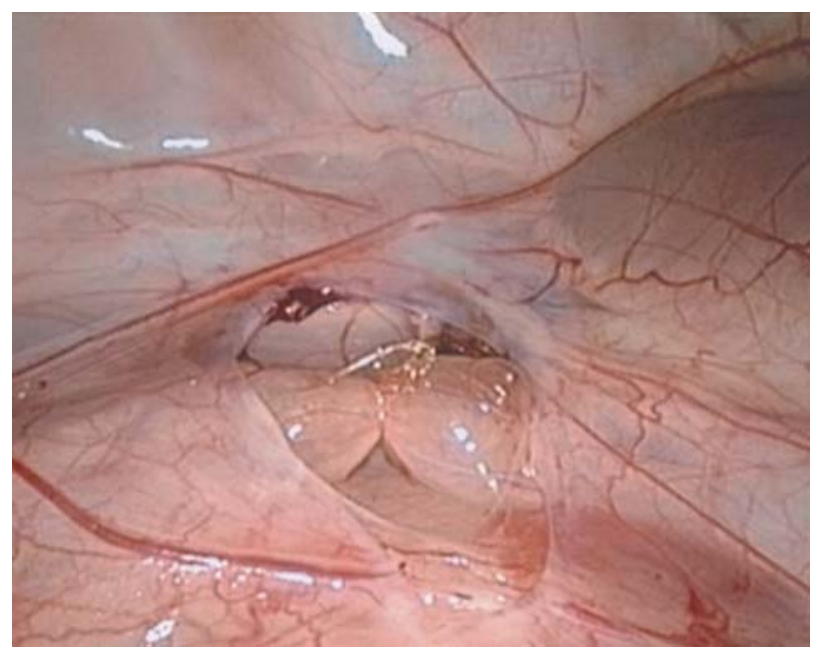

FIGURA 1. Modelo de uropatía obstructiva. neumoperitoneo y se suturan las tres incisiones de los puertos laparoscópicos procediéndose a la recuperación del animal.

\section{Fase II: Diagnóstico y tratamiento de las} estenosis ureterales

Transcurridas 4 semanas de la ligadura ureteral, tiempo necesario para que la sutura (Caprosyn ${ }^{\circledR}$, sutura monofilamento sintética absorbible) se degrade, se procede a la anestesia y posterior extracción de las muestras de orina y sangre. Tras el abordaje transuretral se realizan las mediciones de los mismos parámetros que en la anterior fase. Mediante una RUPG determinamos la longitud exacta y el diámetro ureteral interno. Realizando posteriormente la valoración ecográfica de ambos riñones. La valoración de los ELUS nos permite la confirmación de la uropatía obstructiva y su repercusión en la arquitectura ureteral y la afectación retroperitoneal.

Una vez diagnosticada la uropatía obstructiva, procedemos al tratamiento vía retrógrada de la estenosis ureteral proximal. Los animales son distribuidos al azar en 2 grupos. El grupo I es sometido a una endoureterotomía mediante la técnica de "endoballoon rupture", consistente en la disposición de un balón de dilatación, centrado en la estenosis y su insuflación máxima hasta conseguir una ureterotomía completa de la zona, comprobada mediante la extravasación retroperitoneal de medio de contraste.

$\mathrm{El}$ grupo II formado por los restantes 5 cerdos es sometido a una endoureterotomía mediante el balón con diatermia Acucise ${ }^{\circledR}$. Tras centrar el balón en la estenosis y dirigir el hilo de corte en sentido lateral se activa el Acucise ${ }^{\circledR}$ a 75 vatios de corte puro a la vez que se rellena el balón de dilatación durante 2-4 segundos. Tras 10 minutos el catéter Acucise $^{\circledR}$ es desinflado y retirado. Comprobando de igual modo que en el anterior grupo el éxito de la técnica y disponiendo el mismo tipo de catéter (Fig. 2). En ambos grupos se coloca un catéter ureteral doble pigtail de $7 \mathrm{Fr}$ durante 3 semanas. Los animales son recuperados y hospitalizados.

Tras 3 semanas se retira vía cistoscópica el catéter doble pigtail de ambos grupos. Aprovechando esta circunstancia para la valoración del estado de la vía urinaria. 


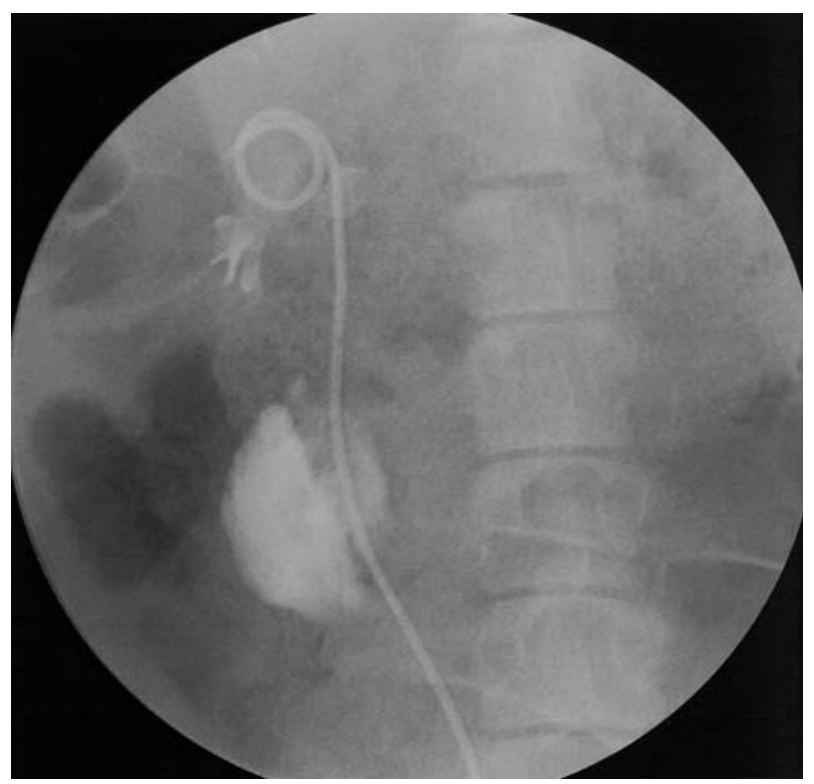

FIGURA 2. Comprobación de extravasación de medio de contraste tras endoureterotomia.

Fase III: Seguimiento y estudios postmortem

Transcurridas 7 semanas del tratamiento de las estenosis ureterales se repite la misma rutina de exploración que en las fases I y II.

Una vez finalizados los estudios de seguimiento y continuando bajo anestesia general, los animales fueron eutanasiados siguiendo las pautas del American Veterinary Medical Association Panel on Euthanasia's guidelines. Procediéndose a completar necropsias de todos los animales. Los riñones, uréteres y la vejiga de la orina son retirados de cada animal en bloque. El segmento ureteral que sufrió la ureterotomía, los riñones y el uréter intramural son seccionados y teñidos mediante las tinciones de Hematoxilina-Eosina, PAS y Van Gienson.

El estudio histopatológico de todas las muestras fue llevado a cabo por el mismo anatomopatólogo (ED) mediante un estudio ciego. Se realiza una clasificación de los hallazgos en la zona de ureterotomía para valorar las lesiones de capa ureteral, según trabajos previos de Kerbl et $\mathrm{al}^{9}$ y Nakada et $\mathrm{al}^{10}$ Tabla 1. Asignando valores entre $(0=$ normal a $3=$ grave $), y$ de este modo creamos una evaluación histológica con valores entre 0 y 18.

\section{Análisis estadístico}

Los resultados experimentales son expresados como media \pm DV (desviación estándar). Los resultados de las medidas del diámetro ureteral interno, la longitud de las estenosis, los valores de la urea y creatinina séricas y la clasificación histológica fueron sometidos a un análisis de ANOVA. En el caso de encontrar diferencias estadísticamente significativas entre las medias de las distintas variables, las diferencias entre medias se establecieron "a posteriori" mediante una prueba de Tukey a un nivel de significación p<.05 (SPSS 10.0 para Windows, SPSS Inc, Chicago,EE.UU).

\section{RESULTADOS}

Fase I

Todos los animales del estudio mostraron ausencia de lesiones tras la valoración sonográfica y mediante urografía excretora del sistema urinario. Los resultados del diámetro ureteral interno en la zona de estudio evidenciados mediante RUPG se muestran en la Tabla 2, no presentándose diferencias estadísticamente significativas entre los 2 grupos de animales respecto a este parámetro antes de la lesión. El recorrido ureteral no muestra codos ni estrecheces antes del modelo experimental de uropatía obstructiva.

Los ELUS en esta fase muestran una conformación normal de todas las capas y ausencia de afectación retroperitoneal, apreciándose a lo largo del recorrido la actividad peristáltica ureteral.

Los valores de la urea y creatinina séricas, se encuentran en todos los animales dentro de los

Tabla 1

Valoración histológica de la zona de ureterotomía

\begin{tabular}{lcccccc}
\hline & $\begin{array}{c}\text { Estado del } \\
\text { urotelio }\end{array}$ & $\begin{array}{c}\text { Inflamación } \\
\text { de la pared }\end{array}$ & $\begin{array}{c}\text { Fibrosis de la } \\
\text { lámina propia }\end{array}$ & $\begin{array}{c}\text { Fibrosis } \\
\text { muscular }\end{array}$ & $\begin{array}{c}\text { Integridad de } \\
\text { la capa }\end{array}$ & $\begin{array}{c}\text { Alteraciones } \\
\text { de la serosa }\end{array}$ \\
\hline GRUPO I & 5 & 6 & 7 & 8 & 8 & $7,66 \pm 0,98$ \\
GRUPO II & 10 & 6 & 6 & 11 & 9 & 5 \\
\hline
\end{tabular}

$\mathrm{p}=0.025$ 
Tabla 2

Diámetros ureterales internos

\begin{tabular}{lccc}
\hline mm & Fase I & Fase II & Fase III \\
Grupo I & $4.84 \pm 0.51$ & $1.86 \pm 0.15^{\mathrm{a}}$ & $4.28 \pm 1.21$ \\
Grupo II & $5.36 \pm 0.66$ & $2.25 \pm 0.20^{\mathrm{b}}$ & $4.56 \pm 0.49$ \\
\hline
\end{tabular}

a: $p=0.0007 \quad b: p=0.002$

rangos normales para la especie porcina. Ningún animal del estudio presentaba reflujo vesicoureteral ni colonización bacteriana de su orina.

Fase II

Transcurridas 4 semanas de la ligadura parcial laparoscópica del uréter todos los animales mostraron signos de uropatía obstructiva del sistema urinario superior. El $70 \%$ de los animales exhiben caliectasia media (grado II de la clasificación de la SFU), 2 animales no muestran prácticamente alteraciones y un animal manifestaba la pelvis agrandada, cálices dilatados y el parénquima renal era normal (grado III).

La UE mostró en los uréteres control ausencia de cambios respecto a la anterior fase. El uréter estenosado valorado mediante esta técnica se aprecia con caliectasia, estasis de la vía urinaria craneal a la estenosis, así como nefrograma persistente.

Mediante la RUPG es apreciable en la práctica totalidad de los animales, dilatación del sistema pielocalicial y aspecto de maza de los cálices renales, uréter dilatado, elongado y tortuoso. La medición del diámetro ureteral interno en la zona de estenosis confirma la reducción de este hasta niveles estadísticamente significativos. Se produce una disminución del diámetro ureteral interno entre la Fase I y la II en ambos grupos, no apreciándose diferencias estadísticamente significativas entre los grupos comparados dentro de cada fase. Tampoco encontramos diferencias estadísticamente significativas respecto a la longitud de la estenosis ureteral en ambos grupos (Tabla 3).

La exploración mediante ELUS del segmento estenosado muestra un manifiesto estrechamiento luminal, con las capas ureterales comprimidas, siendo imposible la diferenciación de estas. Se aprecia en la zona de estudio de todos los uréteres una fibrosis periureteral que rodea por completo
Tabla 3

Longitud de la estenosis en Fase II.

\begin{tabular}{lc}
\hline Fase II & Longitud de la estenosis \\
Grupo I & $4.26 \pm 0.76 \mathrm{~mm}$ \\
Grupo II & $3.26 \pm 0.56 \mathrm{~mm}$ \\
\hline
\end{tabular}

las capas más externas de este (Fig. 3). El segmento ureteral lesionado no evidencia peristaltismo alguno. El segmento craneal a la estenosis se encuentra dilatado con adelgazamiento por compresión de las capas que componen el uréter, por el contrario el segmento distal muestra una apariencia normal.

Los valores de la urea y creatinina séricas no revelan diferencias estadísticamente significativas con la anterior fase en ningún animal. El urocultivo fue negativo en todos los animales del estudio. Ninguno de los animales presentó en esta fase signos de reflujo vesicoureteral.

Tras el estudio diagnóstico se realiza la distribución al azar de los animales para el tratamiento. En un animal de cada grupo se repitió la técnica hasta conseguir la extravasación de medio de contraste al espacio retroperitoneal. De este modo se provocó en un animal del grupo I una gran extravasación de medio de contraste comparado con el resto de animales del grupo.

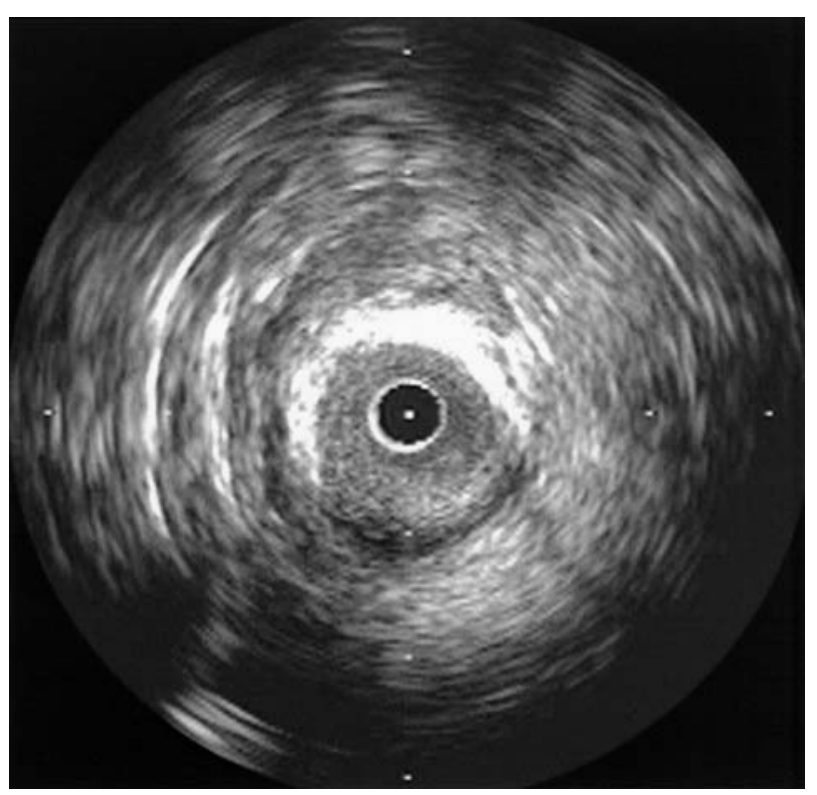

FIGURA 3. Diagnóstico de estenosis ureteral mediante ELUS. 


\section{Fase III}

Los animales de ambos grupos presentan un uréter tortuoso que no ha recuperado la linealidad característica de la Fase I. Mediante la valoración de la RUPG y la ecografía renal, determinamos la desaparición o persistencia de la uropatía obstructiva y el porcentaje de éxito o mejora tras el seguimiento en ambos grupos. En el grupo I encontramos un $80 \%$ de éxito y en el grupo II un 100\%. No existen diferencias estadísticamente significativas entre los diámetros ureterales internos de ambos grupos tras el seguimiento.

El animal del grupo I que no mejora en la Fase III, muestra un grado de hidronefrosis tras su estudio de IV. Esto supone un patrón de uropatía obstructiva más avanzado que en la Fase II.

Mediante el estudio de la ultrasonografía endoluminal apreciamos que las diferentes capas del uréter se diferencian con claridad, mostrando una mucosa regenerada, una capa muscular adelgazada y fibrosada, así como una capa serosa engrosada y con fibrosis más evidente en el grupo I. Todos los animales del estudio muestran una fibrosis periureteral alrededor de la zona de lesión intervenida, evidenciando una fijación del uréter a la musculatura abdominal, más evidente en el grupo I (Fig. 4). El animal con reestenosis mostró una extensa fibrosis de todas las capas

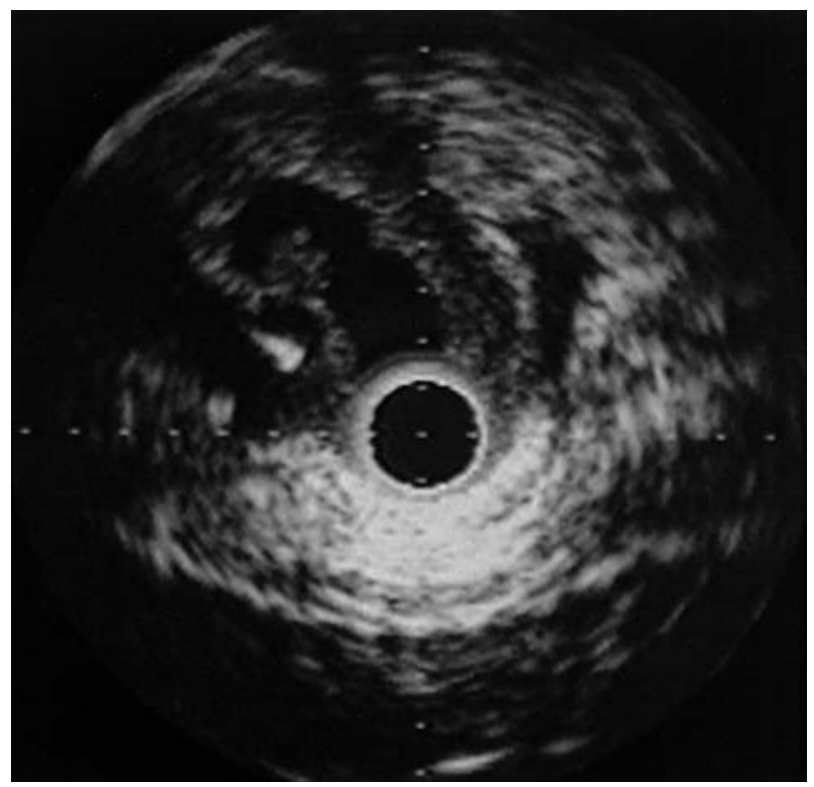

FIGURA 4. Fase III, seguimiento mediante ELUS, grupo I. ureterales, así como del tejido periureteral adyacente. En ningún animal del estudio se recupera el peristaltismo en la zona incidida (Fig. 5).

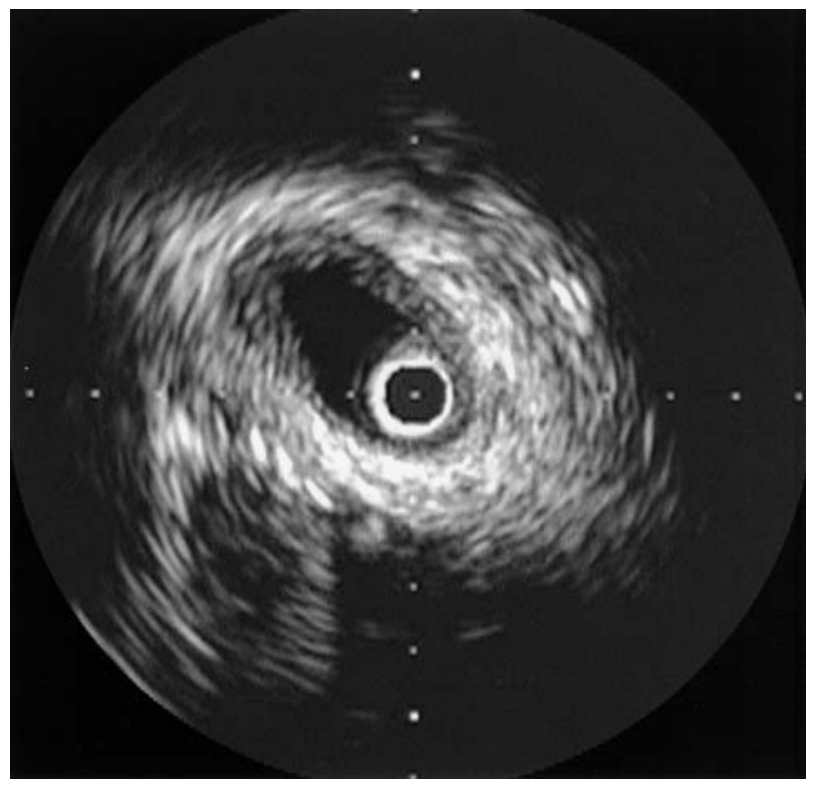

FIGURA 5. Fase III, seguimiento mediante ELUS, grupo II.

La urea y creatinina no presentan diferencias estadísticamente significativas entre ninguna de las fases del estudio. Tras el urocultivo se verifica la presencia de microorganismo en dos animales del grupo II (E. coli, Proteus spp) mientras que las orinas del grupo I eran todas estériles.

La única complicación reseñable es la migración en sentido vesical de un catéter ureteral doble pigtail en un animal del grupo I, siendo este animal el único del estudio que se reestenoso. Coincidiendo este mismo animal con el que en la Fase II presentó una gran extravasación de medio de contraste.

Durante el estudio macroscópico apreciamos adherencias de la zona de ureterotomía al músculo psoas en todos los animales, más patente en los animales del grupo I.

El estudio histopatológico muestra que los uréteres que sufren el modelo de estenosis difieren bastante de lo descrito para los uréteres control. El urotelio se mantiene en dos animales del grupo I, aunque estos presentan varias formaciones quísticas revestidas por epitelio transicional y cuya luz se encuentra ocupada por material PAS positivo. El resto de animales de este grupo muestran un epitelio bien conservado, salvo 
por la presencia de algunas zonas puntuales con ausencia de este tejido. Sin embargo en el grupo II este parámetro de estudio se encuentra más afectado con pérdida de urotelio en zonas más o menos extensas en todos los animales evaluados (Fig. 6A). En otro animal de este grupo II se distinguen pequeñas áreas de hiperplasia urotelial.

La inflamación de la pared es de leve a moderada en ambos grupos. Las células que constituyen los focos inflamatorios son fundamentalmente mononucleares (linfocitos y algunas células plasmáticas) y se localizan en lámina propiasubmucosa, aunque también en la capa muscular y en serosa en grupo I (Fig.6B). La fibrosis de la capa muscular oscila entre el grado leve a severa en ambos grupos. La integridad de la capa muscular sólo se mantiene en un único animal del grupo I y el resto de animales del estudio presentan un intenso fraccionamiento de sus haces musculares (Fig. 6C).

Respecto a las alteraciones de la serosa son manifiestamente más importantes en el grupo I con presencia de extensa fibrosis (Tabla 1). Destacamos finalmente el desarrollo de metaplasia ósea en la lámina propia-submucosa y en la serosa del uréter de un animal del grupo I (Fig. 6D).

Tras el estudio estadístico de las alteraciones histológicas, no encontramos diferencias estadís-

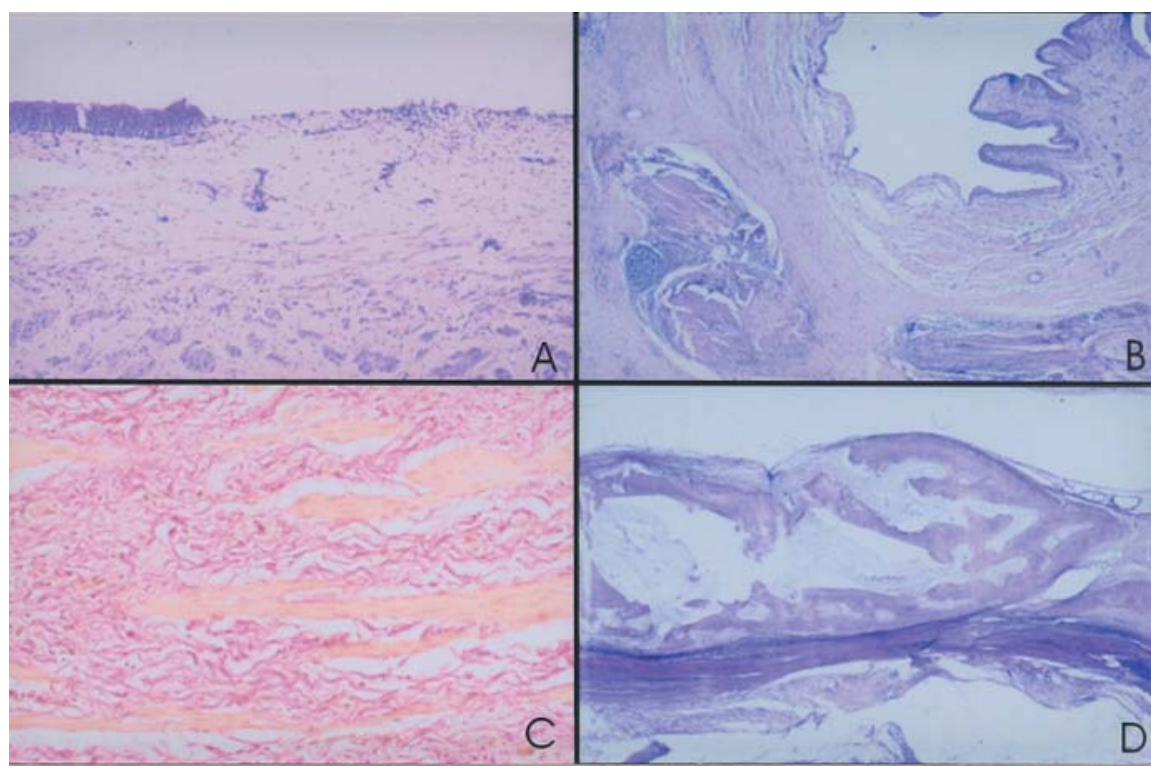

FIGURA 6. A- Zonas carentes de urotelio en zona de estudio, grupo II. B- Foco inflamatorio mononuclear en serosa con presencia de fibrosis. C-Fibrosis, fraccionamiento y atrofia de la de la capa muscular. D-Metaplasia ósea. ticamente significativas entre ambas medias, pero sí en el parámetro de estudio "alteraciones de la serosa" ( $p=0,025)$.

La imagen histológica de los uréteres intramurales de ambos grupos es similar y se acerca claramente a la normalidad diferenciándose únicamente una leve congestión y la presencia de escasos focos inflamatorios mononucleares, constituidos por linfocitos y células plasmáticas, situados en la lámina propia.

\section{DISCUSIÓN}

Durante los último años y debido al gran avance que ha experimentado la cirugia minimamente invasiva, determinadas técnicas han encontrado su hueco en pacientes seleccionados como es el caso de la endopielotomía. Esto no ocurre así con respecto a la endoureterotomía, donde la cirugia convencional continua siendo la técnica de elección ${ }^{6}$.

Un factor de predicción del éxito de la endoureterotomía lo aportan diferentes estudios confirmando que la longitud de la estenosis no debe ser mayor de $2 \mathrm{~cm}^{11}$. Debido a esto en nuestro estudio y gracias al modelo diseñado por Nakada et al no superamos el valor límite de la longitud de la estenosis para decidir su resolución mediante técnicas endourológicas ${ }^{10}$.

Tras la aparición del catéter de balón con electrocauterio (Acucise $^{\circledR}$ ) éste ha ido ocupando un puesto predominante en el tratamiento, tanto de obstrucciones de la unión pieloureteral como de estenosis ureterales $^{12}$. Este sistema es la aproximación más cercana entre la técnica descrita por Davis y la cirugía de mínima invasión para el tratamiento de estas afecciones ${ }^{4}$. Al igual que la endoureterotomía mediante ruptura con balón se inciden todas las capas, comprobando el éxito mediante la extravasación de medio de contraste.

Tras la valoración de la evolución del diámetro ureteral y del grado de hidronefrosis. Consideramos que en nuestro 
trabajo experimental hemos obtenido una tasa de éxito de un $80 \%$ en el grupo I y de un $100 \%$ en el grupo II. En otros trabajos experimentales se obtienen resultados similares para la primera técnica $83 \%-75 \%$ y $67 \%-75 \%$ para la técnica del grupo $\mathrm{II}^{9,10,12}$.

En nuestro estudio la valoración mediante ultrasonografia endoluminal confirma al igual que los posteriores estudios macroscópicos y anatomopatológicos una mayor lesión periureteral y fibrosis retroperitoneal del grupo I, debido posiblemente a que la ureterotomía es más traumática mediante esta técnica. Así como la falta de restablecimiento del peristaltismo ureteral en el segmento ureteral incidido.

El empleo del Acucise ${ }^{\circledR}$ presenta una gran ventaja frente a la endoureterotomía mediante ruptura directa con balón y es la capacidad de control de la ruptura de la estenosis ureteral, consiguiendo siempre incisiones longitudinales de profundidad y amplitud más previsibles. Por contra no se puede predecir el sentido de la ruptura ni la magnitud de ésta mediante la ruptura directa con balón, lo que conlleva en muchos casos a una mayor repercusión retroperitoneal ${ }^{10,12,13}$.

Sin embargo el Acucise ${ }^{\circledR}$, presenta una gran complicación relacionada con las hemorragias graves que se asocian en algunos casos a su empleo $^{14,15}$. Este hallazgo no es nada frecuente en la ruptura directa con balón y en nuestro estudio este riesgo se minimiza al emplear los ELUS en el diagnóstico y seguimiento de los uréteres afectados, evitando de este modo las iatrogenias.

A la vista de los resultados obtenidos en nuestro estudio y tras la comparación con el Acucise ${ }^{\circledR}$, a nuestro parecer la endoureterotomía mediante la dilatación con balón hasta la ruptura ureteral se muestra eficaz en el tratamiento de estenosis ureterales experimentales. La baja difusión de esta técnica se puede deber a lo descrito por Nakada et al: "el miedo que produce una ruptura del uréter en forma de desgarro" 10 , aunque los resultados experimentales sean como mínimo iguales al resto de técnicas endourológicas.

Las ventajas de estas dos técnicas frente a la frecuentemente empleada dilatación simple se deben a que esta última no asegura la ruptura de la capa muscular, causante de la fibrosis que provoca las estenosis ureterales de tipo intrínseco ${ }^{16}$.
Para que la dilatación simple con balón sea efectiva la capa muscular tiene que verse afectada y provocar una posterior cicatrización reparadora que permita mantener esa dilatación ${ }^{17}$. Pero muchos de los resultados de la dilatación con balón implican generalmente sólo hasta la lámina propia y no aparecen afectadas la capa muscular ni la serosa, por lo cual las reestenosis debidas a esta técnica son frecuentes, si no van acompañadas de más acciones sobre el uréter como son la adición de un catéter ureteral o una incisión. De este modo se explican las bajas tasas de éxito de esta técnica, que oscilan entre un $48 \%-88 \%$, con una media de $55 \%{ }^{11}$. La combinación de dilatación con balón y posterior colocación de un catéter ureteral sí aumenta la tasa de éxito de esta técnica ${ }^{18}$.

Los estudios de cicatrización y regeneración ureteral tras la endoureterotomía, describen que todavía se desconoce el mecanismo exacto de la cicatrización ureteral. Tras los estudios de Davis y Oppenheimer et al en la década de los 50, se pensaba que la regeneración de la capa muscular tras la incisión longitudinal del uréter era posible, de este modo se daba base científica a la ureterotomía intubada ${ }^{4,19}$.

Pero en 1992, Schmeller et al, en un estudio histológico de uréteres incididos longitudinalmente mediante endoureterotomía endoscópica, no pudieron demostrar que el hueco después de la incisión se regenerase con tejido muscular, sino que es tejido cicatricial el que ocupa este lugar. El conocimiento sobre la formación de tejido fibroso en lugar de regeneración muscular después de la endoureterotomía no es una contraindicación para este procedimiento ${ }^{20}$. Así ElGammal et al, en un estudio en la especie porcina, concluyen que desconocen si la cicatrización se produce por crecimiento hacia dentro de las fibras musculares desde el labio del área incidida o por regeneración desde fibroblastos pluripotenciales en la zona de cicatriz. Aunque tras sus resultados experimentales sugieren que posiblemente sea una combinación de ambas afirmaciones $^{17}$.

En un reciente estudio experimental Andreoni et al evidencian la presencia de células no epiteliales rellenando el defecto tras una endopielotomía, siendo identificadas como miofibrob- 
lastos y no como células musculares lisas ${ }^{21}$. Al igual que este estudio nosotros encontramos que transcurridas 7 semanas de la ureterotomía el urotelio se ha regenerado y cubre el hueco de la incisión, pero no hay regeneración de células musculares en la zona de incisión. Encontrando en ambos estudios un tejido fibroso junto a fibras musculares fragmentadas y desorganizadas. De este modo no se confirman los hallazgos de los trabajos de Davis ${ }^{4}$ ya que la cicatrización tras las técnicas endourológicas se realiza mediante contracción y no a través de una regeneración de fibras musculares.

\section{CONCLUSIONES}

A la vista de los resultados obtenidos en nuestro estudio las dos técnicas se muestran efectivas en el tratamiento de estenosis ureterales experimentales con porcentajes altos respecto a otras técnicas endourológicas. Aunque el Acucise ${ }^{\circledR}$ supera en \% de éxito a la técnica de dilatación hasta la ruptura ureteral. El empleo del Acucise ${ }^{\circledR}$ presenta una gran ventaja frente a la endoureterotomía mediante ruptura directa con balón y es la capacidad de control de la ruptura de la estenosis ureteral.

No encontramos evidencias histológicas que avalen los estudios de cicatrización de Davis tras la endoureterotomía.

\section{Agradecimientos}

A la Fundación para la Investigación en Urología. A A. Santos, M. Borrega, S. Bravo y B. Loscertales por su importante contribución en este estudio.

\section{REFERENCIAS}

1. Goldfischer ER; Gerber GS. Endoscopic management of ureteral strictures. J Urol 1997; 157(3):770-775.

2. Moon YT; Kerbl K; Pearle MS; Gardner SM; McDougall EM; Humphrey P; Clayman RV. Evaluation of optimal stent size after endourologic incision of ureteral strictures. J Endourol 1995;9(1):15-22.

3. Gruntzig A, Hopff H. Percutaneous recanalization after chronic arterial occlusion with a new dilator-catheter (modification of the Dotter technique). Dtsch Med Wochenschr 1974;99(49):2502-2511.

4. Davis DM. Intubated ureterotomy: a new operation for ureteral \& ureteropelvic stricture. Surg Gynec \& Obstet 1943;76:513.

5. Reimer DE, Coleman O. Iatrogenic ureteral obstruction treated with ballon dilation. J Urol 1981;126(5):689-690.

6. Seseke F, Heuser M, Zöller G, Plothe KD, Ringert RH. Treatment of iatrogenic postoperative ureteral strcitures with acucise endoureterotomy. Eur Urol 2002;42(4):370-375.
7. Soria F, Sánchez FM, Ezquerra LJ, Díaz-Güemes I, Usón J. Diagnóstico y seguimiento mediante ultrasonidos endoluminales del tratamiento endourológico de estenosis ureterales experimentales. Actas Urol Esp 2002;26(3):174-181.

8. Wolf JS Jr, Humphrey PA, Rayala HJ, Gardner SM, Mackey RB, Clayman RV. Comparative ureteral microanatomy. J Endourol 1996;10(6):527-531.

9. Kerbl K, Chandhoke PS, Figenshau RS, Stone AM, Clayman RV. Effect of stent duration on ureteral healing following endoureterotomy in an animal model. J Urol 1993;150(4): 1302-1305.

10. Nakada SY, Soble JJ, Gardner SM, Wolf JS Jr, Figenshau RS, Pearle MS, et al. Comparison of Acucise endopyelotomy and endoballoon rupture for management of secondary proximal ureteral stricture in the porcine model. $\mathrm{J}$ Endourol 1996,10(4):311-318.

11. Hafez KS, Wolf JS. Update on minimally invasive management of ureteral strictures. J Endourol 2003;17(7):453-464.

12. Pearle MS, Moon YT, Endicott RC, Gardner SM, Humphrey P, Clayman RV. Comparison of retrograde endo-pyelotomy and endo-balloon rupture of the ureteropelvic junction in a porcine model. J Urol 1994;152(6):2232-2239.

13. Lewis-Russell JM, Natale S, Hammonds JC, Wells IP, Dickinson AJ. Ten years'experience of retrograde balloon dilatation of pelvi-ureteric junction obstruction. BJU international 2003;93(3):360-363.

14. Preminger GM, Clayman RV, Nakada SY, Babayan RK, Albala DM, Fuchs GJ, Smith AD. A multicenter clinical trial investigating the use of a fluoroscopically controlled cutting balloon catheter for the management of ureteral and ureteropelvic junction obstruction. J Urol 1997;157 (5): 1625-1629.

15. Schwartz BF, Stoller ML. Complications of retrograde ballon cautery endopyelotomy. J Urol 1999;162(5):1594-1599.

16. Fingenshau RS, Clayman RV, Wick MR, Stone AM. Acute histologic changes associated with endoureterotomy in the normal pig ureter. J Endourol 1991;5(4):357-361.

17. El-Gammal M, Hassouna M, Li JS, Wang NS, Coolsaet B, ElHilali MM. Effect of balloon catheter dilatation of the ureter on upper tract dynamics and ureteral wall in swine. J Endourol 1990;4(1):15-26.

18. Snow TM, Wells IP, Hammonds JC. Ballon rupture and stenting for pelviureteric junction obstruction:abolition of waisting is a prognostic marker. Clin Radiol 1994;49(10): 708-710.

19. Oppenheimer R, Hinman F. Ureteral regeneration: Contracture vs. Hyperplasia of smooth muscle. J Urol 1955;74 (4):476-484.

20. Schemeller N, Leitl F, Arnholdt H. Histology of ureter after unsuccessful endoscopic intubated incision. J Urol 1992; 147(2):450-453.

21. Andreoni CR, Lin HK, Olweny E, Landman J, Lee D, Bostwick D, Clayman RV. Comprehensive evaluation of ureteral healing after electrosurgical endopyelotomy in a a porcine model: original report and review of the literature. J Urol 2004;171(2):859-869.

Dr. F. Soria Gálvez

Coordinador de Endoscopia.

Centro de Cirugía de Mínima Invasión

Avd. de la Universidad, s/n

10071 Cáceres

(Trabajo recibido el 9 diciembre de 2004) 